\title{
Problems of drug resistance in tuberculosis- the newer anti-tuberculosis drugs
}

\author{
JOHN CROFTON \\ New Medical Block, City Hospital, Greenbank Drive, Edinburgh, EH10 5SB
}

\section{Summary}

The value of ethambutol and rifampicin is now established but certain points about toxicity and effectiveness are still uncertain, in particular the failure rate if these two drugs are used together without a third.

Primary resistance to ethambutol has been described in the U.S.A. but seems rare in Britain. Acquired resistance to both drugs is now being seen in reference laboratories so that the drugs are already being wrongly used.

It is suggested that, if at all possible, a third drug should be added to the rifampicin-ethambutol combination. The pros and cons of different drugs are discussed.

In future an increasing number of relapsing cases will already have received the new drugs. The clinician will therefore have to work out for each patient the drugs to which his bacilli are probably sensitive. A reliable drug combination for that individual can then be devised.

The principles of such an analysis are outlined and examples given.

IN this brief paper it is not intended to review the newer anti-tuberculosis drugs, ethambutol, rifampicin and capreomycin. General information about these drugs will be familiar. One can assume that ethambutol and rifampicin are extremely useful and effective drugs and that capreomycin is also useful in certain cases. Apart from problems of intermittent chemotherapy there are one or two residual questions such as the following:

(1) With the doses recommended at present is retrobulbar neuritis so rare as to be a negligible risk in the use of ethambutol?

(2) What is the risk of liver toxicity with rifampicin? Are hypersensitivity reactions and the toxic effect on the blood platelets confined to patients in whom the drug has been given intermittently in high dosage?

(3) What is the failure rate in chronic drug resistant tuberculosis if ethambutol and rifampicin are the only drugs used? Is a third drug essential to obtain
$100 \%$ result? It appears that, if both drugs are taken in recommended doses, the failure rate is low but perhaps not negligible.

Up to recently very few patients had received ethambutol and rifampicin and therefore most patients were likely to have sensitive organisms. Primary resistance to ethambutol has been described in the United States (Hobby et al., 1969) but Dr A. T. Wallace tells me that he has not seen it in fairly extensive experience in this country. However strains of bacilli with acquired resistance to bot? ethambutol and rifampicin are now being recorde $\$$ at the Tuberculosis Reference Laboratory at Cardi (Dr J. Marks, personal communication) so the honeymoon period is probably drawing to a closes If these drugs start being used in primary treatment and are not used in the best combination, the number of resistant strains may increase. It seems probable that very shortly one will not be able to prescribe, without further thought, ethambutol and rifampicin for all patients suspected of resistance to the standard drugs and that there will have to be a return to the painful, and not always practised, process of thinking out very carefully the particular problem of each patient with potential drug resistance.

\section{Errors in handling resistance problems}

Common errors are:

(1) Failing to assess in detail the probable resistance pattern in the individual patient on the basis of information about his previous chemotherapy and his reaction to it.

(2) Failure to design for the individual patient a drug combination which is likely to give a very high probability of success.

(3) Failure adequately to handle the psychological problems of the patient so as to persuade him to take the prescribed chemotherapy for the necessary period; these patients are often, quite understandably, cynical about doctors when previously prescribed treatment has failed to control their disease. This made it particularly difficult to persuade patients to take the reserve drugs previously available which 
so often produced subjective symptoms. In this respect, as in others, ethambutol and rifampicin are a marked advance.

\section{Patients who have not previously received ethambutol and rifampicin}

Patients who, on analysis, are likely to have organisms resistant to the standard drugs and/or the previously available reserve drugs should of course be prescribed ethambutol with rifampicin. With present knowledge I would prefer to add a third drug. Capreomycin has been successful in some series but it must be remembered that there can be crossresistance with viomycin or kanamycin. If either of these drugs has been used previously capreomycin should only be relied on as a third drug if the organisms had been shown to be sensitive to it. Prothionamide, if not already used, is another alternative. There may be cross-resistance with thioacetazone, with similar implication as in the case of capreomycin. Moreover, a number of patients are intolerant of prothionamide so that it is wise to avoid it in a patient who is likely to have psychological difficulties in accepting treatment. A third possibility is pyrazinamide, if it has not been previously used. It is a highly effective drug and usually with no subjective side effects but it must be remembered that, like rifampicin, it can have hepatotoxicity so that if both of these drugs are being used liver function should be carefully monitored.

A number of patients who risk having resistance to the standard drugs are likely to have already been treated unsuccessfully with other reserve drugs so that the choice of the third drug to use with ethambutol and rifampicin will depend on the sort of analysis discussed below. In the increasing number of patients who will have had ethambutol or rifampicin, the reliability of each of these drugs for possible further treatment would have to be assessed in the same way. The remainder of this paper will be concerned with such an analysis and some examples of actual patients.

\section{Resistance problems}

Primary resistance to two of the three standard drugs

This is relatively rare but every now and then one finds, after the patient has been on triple chemotherapy with streptomycin, PAS and isoniazid for 2 months or so, that his organisms were primarily resistant to two of the drugs (in my experience usually streptomycin and PAS) before starting treatment. At this stage one does not know whether his organisms have become isoniazid-resistant yet, as he has virtually been having isoniazid alone. One should therefore give an effective combination of reserve drugs, as indicated above, but adding isoniazid in case this has not been lost. At the same time sputum is sent for culture and resistance tests are carried out to isoniazid. If in due course it is found that the organisms are still isoniazid sensitive then this drug can be comined with ethambutol and rifampicin.

\section{Patient presenting with a relapse of tuberculosis}

This may be due to the patient having had a good chemotherapeutic combination but for insufficient time. Up to 18 months of total chemotherapy, the shorter the period of treatment the higher is the risk of relapse. If a good combination has actually been taken, but for too short a period, relapse is likely to occur with sensitive organisms, but if there is any doubt as to whether the patient has taken all the drugs in the combination, or if the disease is advanced, it is safer to give an effective reserve combination but add isoniazid until resistance tests are available.

The patient may have had an unreliable combination of chemotherapy (see below). If there is a relapse in such a patient it should be assumed that the organisms may be resistant to the drugs which had been given. He should receive at least three drugs which he has not had before and to which his organisms are therefore likely to be sensitive. Once more isoniazid should be added if there is any chance that the organisms may still be sensitive to it.

If reserve drugs have also been compromised, the patient has probably had a good deal of chemotherapy. Careful analysis will have to be carried out on the lines indicated below so as to determine to which drugs his organisms are likely to remain sensitive.

\section{Reasons for failure}

(1) A surprisingly common reason for failure is that at some time in the past the patient has been given a drug alone (drug A). Later he either fails to respond to the drug or relapses. The clinician does not realize the risk of resistance to drug $A$ and he treats the patient with $\mathbf{A}+\mathbf{B}$. The patient may therefore be virtually having $B$ alone and may become resistant to it. He later relapses, under treatment or after treatment has been stopped, and is then treated with $\mathbf{A}+\mathbf{B}+\mathbf{C}$. There is clearly a risk of his organisms being already resistant to $A$ and $B$ so that he is virtually having $\mathrm{C}$ alone and is liable to become resistant to $\mathrm{C}$ also.

(2) The patient may have had previous risky chemotherapy such as streptomycin twice or three times a week with daily isoniazid. This carries an appreciable risk of development of resistance first to isoniazid and then to streptomycin. PAS + isoniazid, a two-drug combination, in severe cases may give rise to a failure rate between 10 and $20 \%$. These failures are likely to have organisms resistant to both drugs. Similarly, even in a mild case, if the patient does not take the 
full number of cachets the amount of PAS may be insufficient to prevent isoniazid resistance developing and resistance may emerge first to isoniazid and later perhaps to PAS. Thirdly, with most reserve drugs, at least before the introduction of ethambutol and rifampicin, a combination of only two drugs was liable to lead to a rather high failure rate. Therefore if a patient has been treated with only two drugs and later relapses his organisms may be resistant to these two drugs.

\section{Assessment of individual drugs used}

In the patient who has relapsed, one must therefore ask, of all drugs which have previously been used in that patient, (a) whether the drug was used in a reliable combination, (b) whether any of the companion drugs used were already under risk, because of having been previously used alone or in a risky combination, (c) how long was the drug given: if for more than a few weeks there is risk of resistance if the drug combination was inadequate, (d) what happened to sputum positivity under treatment? If the sputum at first became more negative and later more positive, the patient had either failed to take the drugs or his organisms had become resistant to all the drugs being used at that time. If his sputum had remained positive for more than 6 months on a particular drug combination, this is also highly suggestive that his organisms had become resistant to all the members of the combination. Similarly, radiological deterioration under treatment, if due to tuberculosis and not an intercurrent infection, infarct or carcinoma, is an indication that the organisms have become resistant.

If all these points are carefully assessed the clinician should be able to conclude to which drugs the patient's organisms are likely to be resistant and, in consequence, to which drugs they are likely to remain sensitive. In this way it should be possible to find an effective triple combination for treatment. In many cases it is psychologically undesirable to delay treatment for the long period necessary to carry out resistance tests. Moreover, resistance tests are not reliable in all laboratories.

\section{Examples}

Figure 1 shows an example of a diabetic patient with tuberculous pneumonia who had been treated with streptomycin alone for a month when treatment was stopped owing to the alleged development of deafness. Two years later, as he had become sputum-positive, he had a left thoracoplasty and this was covered by 6 weeks of isoniazid alone. Four years later his sputum became positive again and he had a spread on the other side. He was treated with PAS and isoniazid at home for a period of 2 years. As he had already had isoniazid alone, and in spite
Case 7 (1). J.C., 8,40 , diabetic

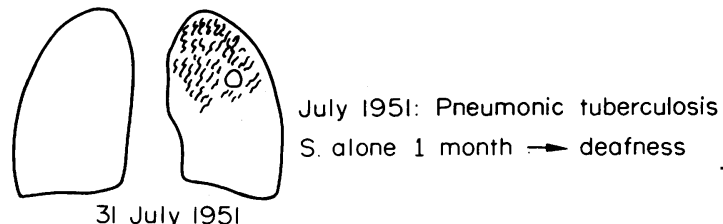

31 July 1951

January 1953: Thoracoplasty

I. alone 6 weeks

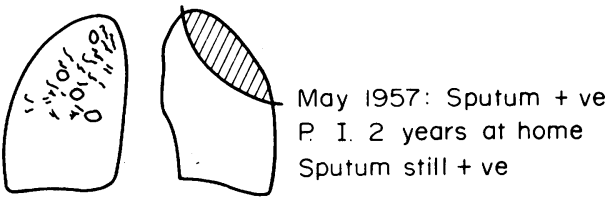

5 May 1957

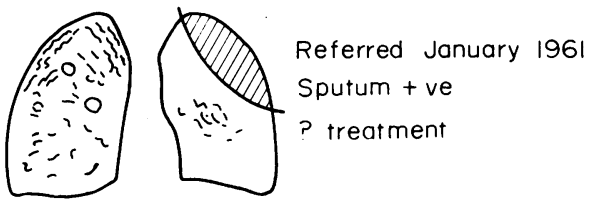

4 Jan. 1961

FIG. 1

of his sputum having become negative after the thoracoplasty, there was a risk that his organisms had become isoniazid-resistant and it was likely that he would then lose PAS. It seemed that he had take. his drugs regularly. The fact that his sputum was stifl positive after 2 years chemotherapy made it virtually certain that his organisms were resistant to botho drugs, either because of previous use of isoniazid alone or because PAS and isoniazid was insufficient $\stackrel{\square}{\varrho}$ to deal with this severe disease. He was referred to $\overrightarrow{\vec{O}}$ me in January 1961 with a further extensive spread 3 of disease on the right side. We assumed that his organisms would be resistant to PAS and isoniazid? and possibly to streptomycin. We designed appro-priate chemotherapy on this assumption and found 3 . in due course that his organisms were indeed resistant to the three standard drugs. Although he had considerable side effects, of one kind or another, with the reserve drugs then available his sputum was converted to negative and remained so.

Figure 2 summarizes a girl aged 13 who was found $>$ to have shadows on the left side of the chest and a을 strongly positive tuberculin test after a routine X-ray. Laryngeal swabs were negative. She was at first watched for several months when there was some 0 radiological deterioration. Laryngeal swabs were stilli negative. She was treated with 'Dipasic', a chemicalo combination of PAS and isoniazid in which theo quantity of PAS is quite insufficient to prevent iso- $\frac{\bar{\Phi}}{\mathbb{Q}}$ niazid resistance. Ten months later there was a fur $\stackrel{?}{?}$ 
Case 8 (1). M.H., 9,13

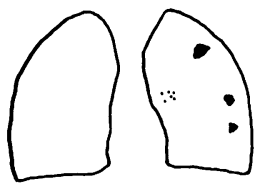

2 Feb. 1955

Observed:

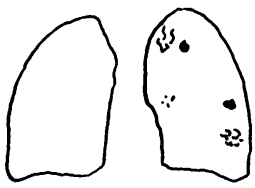

29 July 1955

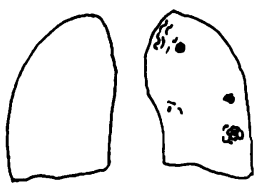

23 May 1956
Routine $X$-ray

Loryngeal swab-ve

Tuberculin + ve

No symptoms

? correct

Laryngeal swab-ve

Treated: Dipasic - several months

Sputum, laryngeal swab-ve

? treatment

Fig. 2

ther radiological deterioration. This might be because she had only had a few months' treatment or might be because her organisms had become isoniazid resistant. Both possibilities could have been correct. Figure 3 shows her further progress. She was treated with PAS and isoniazid for 5 months. If her organisms had become isoniazid-resistant there was therefore a possibility that they might now have become PASresistant. As there was further radiological deterioration streptomycin was then added, which carried the risk of the development of streptomycin resistance. Laryngeal swabs, however, were still negative. There followed a fairly long period of treatment either with all three standard drugs or with PAS and isoniazid but at the end of that time there was further radiological deterioration and her laryngeal swabs became positive for the first time. She was referred to me. analysis made it likely that her organisms were resistant to the three standard drugs and this proved to be correct. As we had only limited reserve drugs at that time she was treated with these and with resection and with success. She now has two children and is very well.

The last patient had an extensive story of chemotherapy (Fig. 4). I was asked to advise about him in 1965 by which time he had had resection of his left upper lobe with a thoracoplasty. The whole of his left lower lobe was shown in due course to consist of a single enormous cavity. He had extensive disease in the upper two-thirds of his right lung. In 1950 he had had PAS alone for 7 months and his sputum had become negative. A year later his sputum had become positive again and he was treated with streptomycin and PAS. If his organisms had become re-
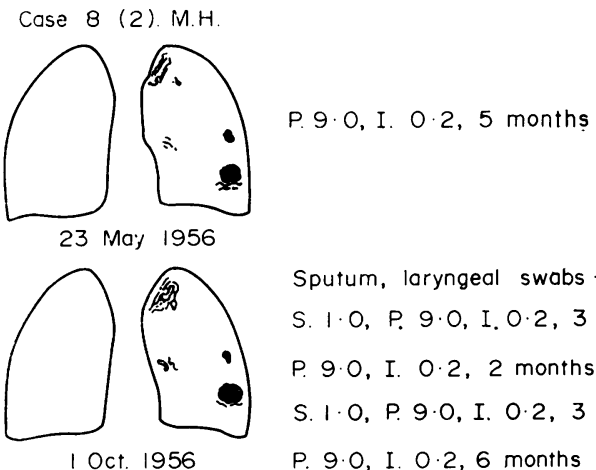

Sputum, laryngeal swabs - ve

S. $1 \cdot 0$, P. $9 \cdot 0$, I. $0.2,3$ months

P. $9 \cdot 0, I .0 \cdot 2,2$ months

S. $1 \cdot 0$, P. $9 \cdot 0$, I. $0.2,3$ months

P. $9 \cdot 0,1 \cdot 0 \cdot 2,6$ months

S. I. O, P. $12 \cdot 0$, I. $0 \cdot 25,6$ months

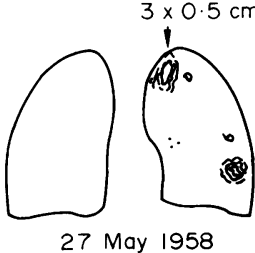

Laryngeal swab $+v_{\theta}$

Sensitivities unknown

? treatment

Fig. 3

sistant with the previous mono-therapy, in spite of the sputum having become negative, there was obviously a risk of losing streptomycin. However, once more his sputum became negative on the double combination. Six years later the sputum became positive again and he was treated with daily streptomycin and isoniazid. In due course it was found, as obviously might have been guessed from his previous treatment, that his organisms had become resistant to streptomycin, PAS and isoniazid. He was then treated (Fig. 5) with viomycin $2 \mathrm{~g}$ twice a week combined with high doses of tetracycline and with isoniazid for 8 months. This was followed by tetracycline and isoniazid for 9 months after which his sputum was still positive. In 1959 he was given pyrazinamide and cycloserine for 6 months. This double combination has a fairly high failure rate.

Case 10 (1). A.A., \%, 48

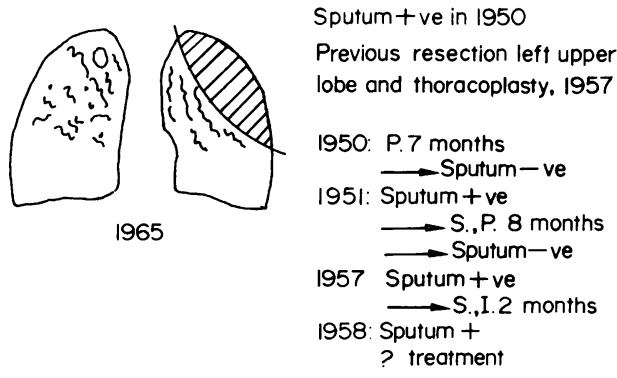

FIG. 4 


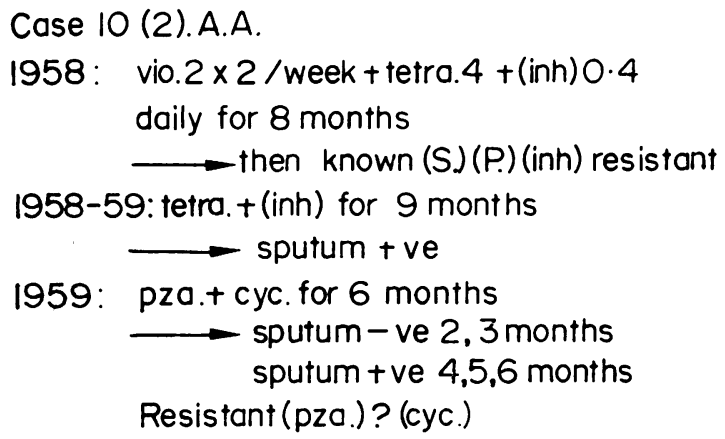

FIG. 5

Fig. 6

His sputum was negative at the second and third months of treatment but positive at the fourth, fifth and sixth months, indicating that his organisms had become resistant to both drugs. Further progress is shown in Fig. 6. Ethionamide had then become available and he was given it with viomycin and cycloserine. Unfortunately previous treatment had made it likely that his organisms were resistant to both of the latter two drugs. After a year his sputum had again become positive and he was treated with viomycin and thioacetazone. There is liable to be crossresistance between thioacetazone and ethionamide. The fact that the sputum had been positive after a year of ethionamide made it virtually certain that his

organisms had become resistant to that drug. He then had various combinations of drugs to which his organisms were almost certainly resistant and in 1965 his sputum was still positive. It was in fact found that his organisms were still sensitive to kanamycin. He was treated with kanamycin, rifamycin and ethambutol, later, when it became available, with rifampicin instead of rifamycin. His sputum became negative and he was doing very well but unfortunately, about the fifteenth month of treatment, he developed pneumonia in his one normal bit of lung and died. Numerous cultures at post-mortem were all negative.

\section{Conclusions}

The honeymoon period with ethambutol and rifampicin is probably drawing to a close. These drugs are already being used badly in some patients and resistance to them is beginning to emerge. In any case, a third drug is probably best given with these two, even if the patient's organisms are sensitive to both of them. When the clinician is presented with a patient with relapse of tuberculosis he has an important responsibility to analyse very carefully the patient's chemotherapy. Careful analysis can usuall $\bar{b} \mathbf{z}$ indicate to which drugs the patient's organisms are resistant and in consequence to which drugs they are likely still to be sensitive. In this way it is possible t\& design an effective drug combination which wip render his sputum permanently negative. Even i⿺ patients whose organisms are resistant to the stant. dard drugs $100 \%$ success in the arrest of the diseaser and the sputum conversion is now a reasonable aim and the clinician should be satisfied with nothing less.

\section{Reference}

Hobby, Gladys L., Johnson, Peggy M., Boytar-PapirnYik, Valeria, \& Wilber, Judith (1969) Primary drug resistance: A continuing study of tubercle bacilli in a veteran population within the United States. VI. Initial observations on the incidence of resistance to rifampicin and ethambutol. American Review of Respiratory Diseases, 99, 777. 